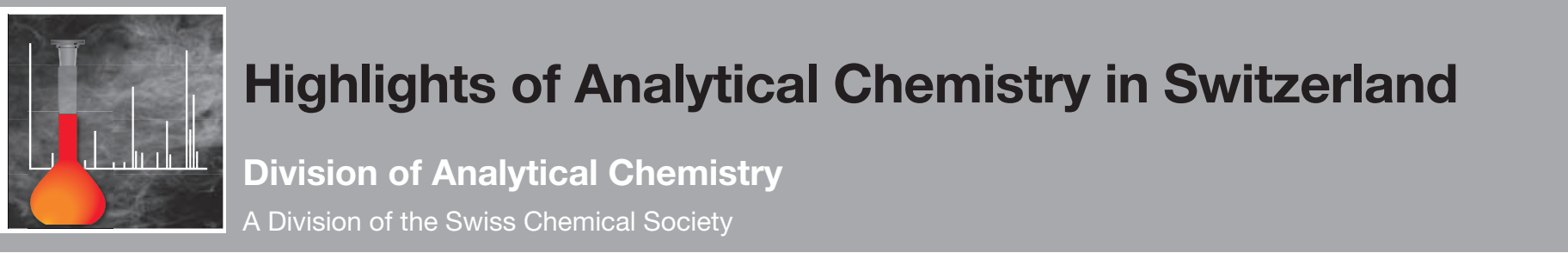

\section{Continuum Between Ritual and Medicinal Use of Plants: Smoke Analysis of Ritual Plants from Southwest China}

Caroline S. Weckerle*, Peter O. Staub, and Florian P. Schiestl

${ }^{*}$ Correspondence: C. S. Weckerle, Institute of Systematic Botany, University of Zurich, Zollikerstrasse 107, CH-8008 Zurich, Tel.: +4144634 83 52, Fax: +41 4463484 03, E-mail: caroline.weckerle@systbot.uzh.ch

Keywords: Ethnobotany · Headspace method · Incense · Smoke analysis · Southwest China · Volatile organic compounds

Plant-derived smoke is used in diverse cultures and regions of the world for various purposes, e.g. as medicine, incense, for food conservation or as insect repellent. Around 1500 plant species are known to be used to produce smoke for various uses. Beside frankincense and myrrh, only little research has been conducted on plants burnt for their fragrant fumes. It remains largely unknown which compounds of the different smokes are responsible for the various impacts smoke may have.

In Southwest China, especially in the Tibetan cultural area, the use of ritual and incense plants is very common. Incense plants are burned freshly or dried and are used to communicate with deities and spirits of the surroundings but also for the treatment of diseases such as respiratory ailments. Our research focuses on a comparative analysis of ritual plants used among different ethnic groups in this area. Since we are especially interested in the continuum between ritual and medicinal use of incense plants and aim to understand the rationale for their use as medicine, we look at the cultural context of plant use, but also analyze the volatile organic compounds (VOCs) of the smoke. So far we documented some 40 ritual plants. Foremost among the incense plants are Juniperus, Cupressus, and Rhododendron species. Important characteristics of incense plants are the density,

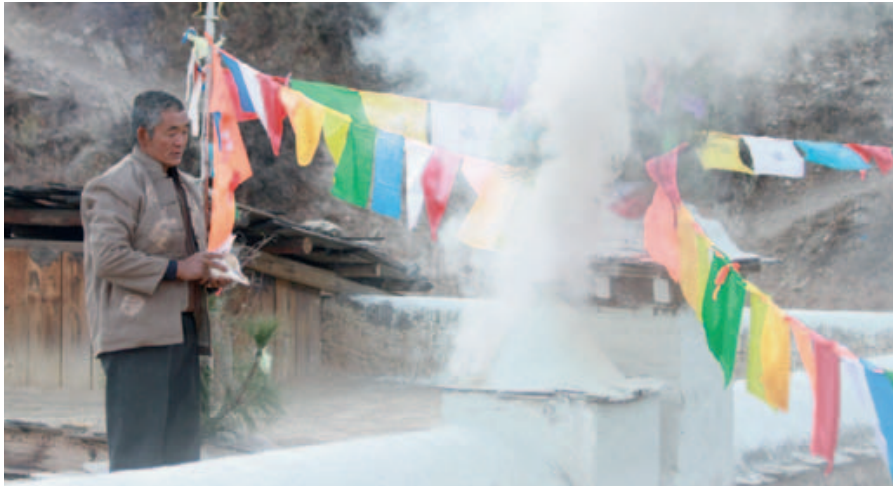

Tibetan man worshipping the mountain gods. He burns incense plants in a ritual burner on the flat roof of the house.

color, and smell of their smoke as well as their habitat. The higher up in the mountains and hence the closer to the mountain gods the plant grows, the more effective it is thought to be.

To collect VOCs of incense smoke we have used the dynamic headspace sorption method. This technique is simple to use, and smoke samples can either be collected directly in the field or from burnt dry plant material in the lab. In this method, VOCs are trapped onto an organic polymer (e.g. Porapak Q), eluted with solvent after collection, and subsequently analyzed using coupled gas chromatography-mass spectrometry (GC-MS). Smoke samples collected and analyzed in this way contained many typical plant secondary metabolites, such as mono- and sesquiterpenoids, benzenoids, and methoxylated phenolic compounds.

Based on the analysis of volatile organic compounds of the smoke and the cultural meaning of ritual plants, hypotheses for their successful use in health-related issues will be developed.

Received: April 13, 2011

Reference

C. S. Weckerle, F. K. Huber, Y. P. Yang, W. B. Sun. Economic Botany 2006, 60, 3 .

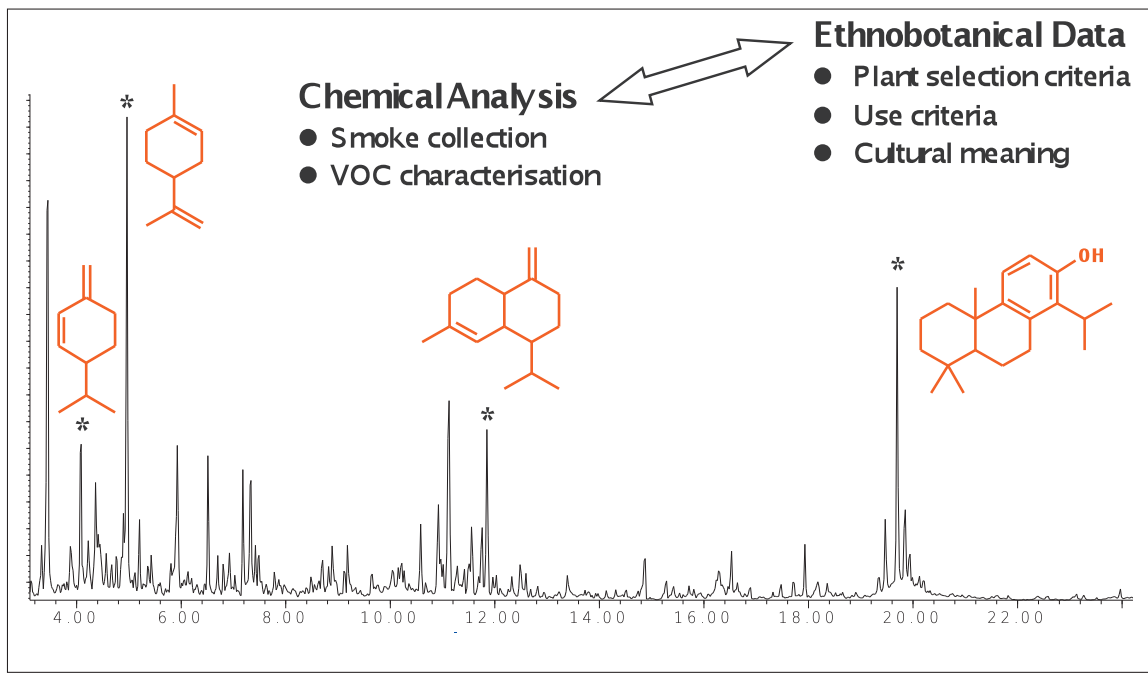

We look at the cultural context of incense plant use in Southwest China and analyze the volatile organic compounds of the smoke, in order to investigate the continuum between ritual and medicinal use of incense plants. Gas chromatogram of the smoke of Cupressus funebris. The peaks marked with an asterisk are, with increasing retention time: $\beta$-phellandrene, limonene, cadinene, and totarol. Their chirality was not investigated. 